\title{
The post-take ward round pro forma: more than a blank space
}

\author{
Authors: Ketan Patel, Helena Roth and James Wilkinson
}

\section{Aims}

To redesign the post-take ward round (PTWR) pro forma to improve clarity and documentation of the PTWR.

\section{Methods}

We obtained feedback from multiple sources and found that the existing pro forma did not standardise documentation of the PTWR. Due to differing styles of the clinicians leading and documenting the PTWR there was a vast variation in the documentation of the PTWR. This meant that key information was not documented which was felt to have a negative impact on patient care.

Given the above, we redesigned the pro forma to provide clear prompts for the junior doctor to ensure key details are documented on the PTWR. The existing pro forma consisted of large blank spaces and no prompts for key information to be documented.

The redesigned pro forma was piloted for a 1-month period and its use was audited and compared against the existing pro forma.

We audited and compared documentation for the following criteria: Time, Date, Observations, Escalation Plan, Working diagnosis, Clinician details.

\section{Results}

We audited 99 PTWR entries of which 49 were using the redesigned pro forma and 50 were using the existing pro forma.

Overall, documentation attainment of the set criteria was improved using the redesigned pro forma when compared against the existing pro forma. The new pro forma averaged a $76 \%$ attainment of the criteria, while the existing pro forma only managed an average attainment of $42 \%$.

One particular aspect of note was the improvement in documentation of the clinician's details. Only $38 \%$ of notes using the existing pro forma had documented name and contact details of the junior doctor compared with the $89.7 \%$ using the redesigned pro forma.

Documentation of escalation plan was very poor with the existing pro forma with only $2 \%$ of notes having any documentation regarding ceiling of care, this improved to $32 \%$ with the redesign. Although an improvement, the attainment should be greater and we will explore further why documentation of this particular criteria is still poor.

\section{Conclusion}

Current Royal College of Physicians (RCP) guidelines recommend a page in admission documentation to be titled 'post-take ward round', however, there is no clear guidance on its design and layout. As shown in this project a blank space titled PTWR is not enough.

The document needs to have prompts for the clinician documenting the PTWR and be organised such that key details such as diagnosis and ceiling of care are easily visible.

We discovered that despite having a prompt regarding 'ceiling of care' this was not documented well. We felt that this was due to a combination of both clinician attitudes about making decisions regarding ceiling of care and the prompt not being clear enough. We will explore this further with our next redesign.

The importance of the PTWR is that it is the first senior review a patient has following admission and its documentation is pivotal as this will often guide other doctors who care for the patient for the remainder of their admission. Through this project we have shown that the layout of the PTWR is important in ensuring clear documentation and can ultimately improve the patient's treatment pathway and outcome. 